\title{
The Application of $\mu$-Law Companding to Mobile WiMax
}

\author{
Brian G Stewart ${ }^{1}$ and Athinarayanan Vallavaraj ${ }^{2}$ \\ ${ }^{1}$ Glasgow Caledonian University \\ Scotland, UK \\ ${ }^{2}$ Caledonian College of Engineering \\ Sultanate of Oman
}

\section{Introduction}

The IEEE802.16e mobile WiMax standard employs Orthogonal Frequency Division Multiplexing (OFDM) principles in the transmission of data (IEEE802.16e, 2005). Within multicarrier systems, like WiMax and other OFDM technologies, a major problem relates to issues associated with instantaneous values of the peak transmission output power. At some instant in time, the subcarriers of an OFDM signal may add coherently producing a very high peak power that may reach a maximum value of the number of subcarriers times the average power. The peak power can be expressed in relation to the average power, referred to as the Peak-to-Average Power Ratio (PAPR), which is defined as the ratio of the peak of the instantaneous envelope power to the average power of the OFDM signal. One of the main drawbacks of WiMax systems is the high value of PAPR often encountered, typically around levels of $12 \mathrm{~dB}$ to $13 \mathrm{~dB}$ or even higher (e.g. Lloyd, 2006). A high PAPR necessitates that the A/D and D/A converters used in the communication system have a higher level of bit conversion to accommodate the peaks. In addition it requires the OFDM power amplifiers to remain linear over an extended region above the average power value to include the peak amplitudes. Also, if there are any regulatory or application constraints on the extent of peak power, a high PAPR would require the average power of the signal to be reduced, thus reducing the range of transmission of OFDM signals (Han \& Lee, 2005). The nonlinearity of any power amplifiers also introduces in-band and out-of-band radiation or spectral splatter, increasing the Bit-Error-Rate (BER) and causing interference with neighbouring frequency channels.

A variety of techniques have been published in the literature which attempt to reduce the PAPR in OFDM signals. These techniques can be classified into three broad categories as, signal pre-distortion techniques, coding techniques and scrambling techniques (Van Nee \& Prasad, 2000). There are also techniques that combine either two or more of these techniques in order to improve the PAPR reduction. Though many solutions have been proposed to deal with the high value of PAPR existing in random data transmission within OFDM systems, one method of PAPR reduction which has received little critical attention in this area is the application of companding. In an attempt to address this weakness, this chapter 
presents a thorough investigation of the performance of $\mu$-Law companding to mobile WiMax and in particular to the Down Link Partially Used Subcarrier (DL PUSC) mode of operation. Parameters investigated and quantified as a function of various $\mu$-Law companding profiles include the Power Spectral Density (PSD), BER, PAPR reduction, and the influence of mobility on performance when WiMax multipath mobile channels are considered. Many of these results are new and have never been investigated for companding or specifically evaluated in relation to WiMax architectures. One further aspect presented in this chapter, which is often neglected in the literature, is the comparison and evaluation of companding in regard to equalised symbol power for all companding situations. It is well known that companding naturally increases the average power of OFDM symbol transmissions. However, equalised symbol power transmission performance requires to be quantified fully to allow a complete understanding of the limitations of companding within WiMax systems. Results will show that companding does have potential for application to mobile WiMax, but there are limitations in relation to PSD, BER, PAPR reduction and mobility, and these will be discussed within the relevant sections.

The structure of the chapter is as follows. Section 2 introduces the concepts and definitions associated with PAPR. Section 3 briefly discusses the general techniques which are currently employed to reduce the PAPR of OFDM data symbols; Section 4 introduces the principles associated with companding and in particular $\mu$-Law companding; Section 5 presents details of the mobile WiMax physical layer model used for the simulations and investigations; Section 6 discusses the issues of PSD related to WiMax companding; Section 7 investigates the BER performance; Section 8 presents the PAPR improvements and Section 9 investigates the influence of mobility for companded WiMax within two common multipath channels. Section 10 is a conclusions section and summarises the main points from the chapter.

\section{The PAPR of an OFDM Signal}

The instantaneous amplitude of a baseband OFDM signal can be written as

$$
x(t)=\sum_{n=0}^{N-1} X_{n} \exp j\left(\Phi_{n}+2 \pi n t / T\right)
$$

where $X_{n} \exp \mathrm{j}(\Phi)$ is the complex baseband modulated symbol, and $N$ is the number of subcarriers. The instantaneous envelope power of an OFDM signal, assuming a unity impedance load, is evaluated through

$$
P(t)=|x(t)|^{2}=\sum_{n=0}^{N-1} X_{n}^{2}+2 \sum_{n=0}^{N-2} \sum_{m=n+1}^{N-1} X_{n} X_{m} \cos \Psi_{n m}
$$

where $\Psi_{n m}=\left(\Phi_{n}-\Phi_{m}+2 \pi(n-m) t / N\right)$. The average envelope power is calculated through

$$
P_{\text {avg }}=E\left\{|x(t)|^{2}\right\}
$$

where $E\{$.$\} is defined as the expectation value. Using equation (1), the expression for the$ average power becomes 


$$
P_{\text {avg }}=E\left\{|x(t)|^{2}\right\}=\sum_{n=0}^{N-1}\left|X_{n}\right|^{2}+\sum_{n=0}^{N-2} \sum_{m=n+1}^{N-1} E\left(X_{n} X_{m}^{*}\right) \exp j(2 \pi(n-m) t / T)
$$

The symbols on different subcarriers within OFDM may be assumed to be independent, and hence, $E\left(X_{n} X_{m}{ }^{*}\right)=E\left(X_{n}\right) E\left(X_{m}^{*}\right)$. Since the signals are orthogonal, then the second term in (4) is zero thus the average power reduces to

$$
P_{\text {avg }}=E\left\{|x(t)|^{2}\right\}=\sum_{n=0}^{N-1} X_{n}^{2}
$$

Since PAPR is defined as the ratio of the maximum (peak) instantaneous envelope power to the average power, then the PAPR may be expressed as

$$
P A P R=\max \left\{\frac{P(t)}{P_{\text {avg }}}\right\}=\max \left\{\frac{|x(t)|^{2}}{E\left\{|x(t)|^{2}\right\}}\right\}
$$

Substituting (2) and (5) into (6) results in the general formula for the PAPR of a general MQAM OFDM transmission, i.e.

$$
P A P R=\max \left\{1+\frac{2}{\sum_{n=0}^{N-1} X_{n}^{2}} \sum_{n=0}^{N-2} \sum_{m=n+1}^{N-1} X_{n} X_{m} \cos \Psi_{n m}\right\}
$$

To help appreciate PAPR, consider MPSK modulation where the amplitudes of all the baseband signals are equal. In this situation equation (7) reduces to

$$
P A P R=\max \left\{1+\frac{2}{N} \sum_{n=0}^{N-2} \sum_{m=n+1}^{N-1} \cos \left(\Phi_{n}-\Phi_{m}+2 \pi(n-m) t / T\right)\right\}
$$

If the data symbols are presumed to be identical on all subcarriers, then when $N$ subcarriers are added together with the same phase, they sum up coherently and produce a peak power that is $N$ times the average power. Figure 1 illustrates the ratio of the instantaneous envelope power to the average power of a single OFDM symbol transmission of period $T$ which comprises 16 QPSK subcarriers all carrying the same data. For this situation, the output from the IFFT produces a single peak at the first and last of the 16 time sampled points of the symbol with zero at all other time samples. The maximum value of the envelope power to the average power (i.e. the PAPR) is $16(=12.04 \mathrm{~dB})$, indicating that the peak power is 16 times greater than the average power. In most cases the PAPR situation to be addressed relates to random data and methods used to reduce PAPR in these situations are briefly discussed in the next section. 


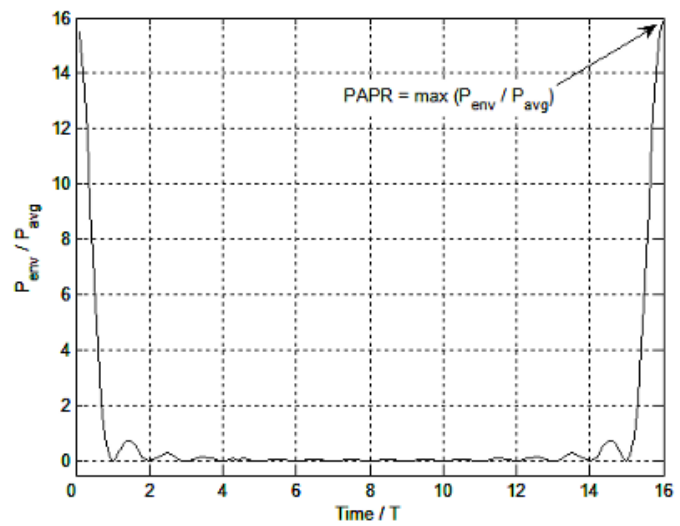

Fig. 1. The normalised instantaneous power transmission for a 16-subcarrier QPSK OFDM symbol when the data on each subcarrier is identical

\section{Reducing the PAPR of OFDM Signals}

\subsection{Methods of PAPR Reduction}

A number of PAPR reduction techniques that attempt to reduce the maximum PAPR of random data within OFDM signals exist (see for example the reviews by Han \& Lee, 2005, and Wang \& Tellambura, 2006). The most popular of these are signal pre-distortion techniques such as clipping, peak windowing and peak cancellation which aim to reduce the peak amplitudes of the transmitted signals by non-linearly distorting the OFDM signal at or around the peak values (e.g. O'Neill \& Lopes, 1995; De Wild, 1997; Li \& Cimini, 1997; Pauli \& Kuchenbecker, 1997; May \& Rohling, 1998; Van Nee \& De Wild, 1998; Van Nee \& Prasad, 2000; Armstrong, 2001, 2002; Wang \& Tellambura, 2005)

A second category of PAPR reduction techniques relates to probabilistic and scrambling methods comprising phase modification techniques, amplitude modification techniques and scrambling and interleaving techniques. These are becoming perhaps the most popular methods of reducing the PAPR in data transmissions within OFDM systems. All these techniques modify the phase, amplitude or subcarrier position of input symbols, thus creating several OFDM signals representing the same information. The OFDM signal with the lowest PAPR is then selected for transmission (e.g. Boyd, 1986; Bäuml et al. 1996; Van Eetvelt et al., 1996; Müller \& Huber, 1997a, 1997b; Cimini \& Sollenberger, 2000; Hill et al. 2000; Jayalath \& Tellambura, 2000; Breiling et al., 2001; Tellambura \& Jayalath, 2001; Han \& Lee, 2005; Wang \& Tellambura, 2006). In most cases extra overhead or side band information is also required to be sent to allow recovery of the original information at the receiver. Perhaps the best known and most popular of these techniques are called Selected Mapping (SLM) and Partial Transmit Sequences (PTS).

A third category of PAPR reduction methods relates to coding techniques. Block and channel coding, or specialised codewords with particular and special autocorrelation properties are employed in an attempt to reduce the PAPR. One of the additional advantages of these techniques is that improved BER as well as reduced PAPR can ensue though at the cost of increased redundancy (e.g. Golay, 1961; Jones et al., 1994; Jones \& 
Wilkinson, 1995, 1996; Davis \& Jedwab, 1999; Paterson \& Tarokh, 200; Tarokh \& Jafarkhani, 2000; Breiling et al., 2001; Yang \& Chang, 2003, Han \& Lee, 2005; Kang, 2006). Often though, significantly reduced PAPR cannot always be guaranteed for all symbol transmissions using these techniques. However, developments and refinements of these techniques are constantly being investigated and reported.

\subsection{Choice of PAPR Reduction Techniques}

Pre-distortion techniques like clipping and filtering are the simplest to implement and do not require any side information to be transmitted, however they result in a distorted signal which produces in-band and out-of-band signal splatter. Peak cancellation, however, does not result in any frequency signal splatter. Scrambling and probabilistic techniques, such as SLM and PTS, are distortionless methods. The complexity however of these techniques is increased in that the number of IFFT operations increases in proportion to the number of scrambled sequences used to produce a reduced PAPR. In addition, these techniques, in general, need side information and as a result the data rate is decreased. There may also be a small compromise on the PAPR due to the transmission of this side information. Coding techniques increase the complexity of the PAPR reduction solution with an additional requirement of encoding and decoding at the transmitter and receiver. As encoding increases the number of bits in the transmitted signal, the data rate is therefore reduced. There is no distortion or signal splatter as in clipping, and encoding can also serve the dual purpose of BER reduction and PAPR reduction.

Clearly there are a variety of PAPR reduction techniques available with each one claiming to have some advantages over the other. The choice of a particular technique depends on a number of factors, for example, PAPR reduction capability required, PSD distortion, acceptable BER at the receiver, signal power requirements, data rate employed, implementation complexity, consideration of the effect of the components in the transmitter, etc. Han \& Lee (2005) have outlined a brief description of these criteria. The quest for inventing new PAPR reduction techniques has not come to an end. With the increasing use of OFDM in mobile broadband applications, the necessity for PAPR reduction has gained critical importance since an increased PAPR means an increased envelope power and thus a reduction in battery standby and battery life time.

One other method of reducing PAPR is called companding. This method falls best under the category of pre-distortion technique. A limited number of publications exist on companding. These publications indicate that companding may have potential in reducing PAPR, but this potential has still to be fully explored and quantified for OFDM type systems. In this regard, an evaluation of companding for Mobile WiMax forms the main thrust of this chapter. The method of $\mu$-Law companding will be introduced in the next section.

\section{Companding of OFDM Signals}

Companding is fundamentally the process of compressing amplitude signals at a transmitter and expanding them at a receiver. A number of authors have advocated the use of companding techniques to OFDM systems to improve the PAPR. Wang et al. (1999) introduced companding as a potential PAPR reduction technique and provided the transmitted waveforms of 16QAM based 256-subcarrier OFDM signals before and after 
companding. The symbol-error-rate (SER) was also shown to vary with the companding coefficients. However, no quantified results in terms of precise PAPR reduction or SER improvement as a function of companding parameters were detailed. Huang et al. (2001) demonstrated that a non-linear-quasi-symmetrical $\mu$-Law companding transform can outperform a clipping-filtering scheme by $4.6 \mathrm{~dB}$ in relation to SNR for a BER of $10^{-4}$ in an additive white Gaussian noise channel, and a PAPR reduction of $4.1 \mathrm{~dB}$ could be achieved for QPSK based 128 subcarrier OFDM signals. Companding profiles considered have included traditional $\mu$-Law and A-Law, as well as exponential type forms (e.g. Jiang \& Song, 2005). Companding of OFDM has also normally been restricted to situations where no pilots are included, one type of modulation is employed, and smaller numbers of subcarriers are considered (e.g. Vallavaraj et al., 2004). The literature therefore demonstrates that companding may be considered to have some validity in relation to possible PAPR reduction. However, one of the main drawbacks of companding is that as a consequence of the non-linear companding profile, PSD distortion occurs resulting in frequency splatter where residual frequency power is "splattered" out with the transmission bandwidth causing inter channel interference. Spectral re-growth also occurs within the OFDM channel bandwidth as a consequence of increased power arising from the companding process. This increased power is also considered to provide an advantage of improved BER due to the effective increased SNR naturally arising from the direct application of companding (Mattsson et al., 1999). However, the question of how spectral re-growth and distortion effects precisely influence the quantification of the performance of an OFDM system has still to be fully considered for OFDM architectures. These issues will be explored in more detail for companded WiMax in the following sections.

\subsection{The Concepts of Companding}

Companding is a very popular technique in communication engineering, especially in voice communication systems using Pulse Code Modulation (PCM) (e.g. Lathi, 1998). A PCM block consists of a signal sampler, an amplitude quantization unit and an encoder. The quantization process leads to the approximation of the amplitudes of the samples. Considering a quantization step size of $Q$, the amplitude of a sampled signal that falls into this particular step size level will be represented by the quantization value of this level irrespective of the actual amplitude of the sample. This process introduces a maximum quantization error of $\pm \mathrm{Q} / 2$.

The quantization error is the difference between the quantized output value and the true value of the sample. Quantization error adds noise to the signal, known as quantization noise. Generally, as the size of all quantization steps is the same, the quantization error will be constant for all steps thus the quantization noise is constant, while the signal amplitude can vary. This results in a varying signal-to-quantization noise power ratio, SQR, given by

$$
S Q R=\frac{\text { Signal Power }}{\text { Quantisation Noise Power }}=\frac{P_{s}}{P_{q n}}
$$

As $P_{q n}$ is constant, the SQR is directly proportional to the signal power $P_{s}$, which means that the large signals will have a higher $\mathrm{SQR}$ and hence a better quality than the small signals. The SQR can be maintained constant if $P_{q n}$ is decreased or increased in the same proportion as the decrease or increase of $P_{s}$. 
The process known as companding in PCM systems is used to maintain a constant SQR. The signal to be transmitted is passed through an amplifier with a non-linear transfer characteristic that favours amplification of the small-amplitude signals. The signal then appears large during quantization and hence the effect of quantization noise upon smaller signals is reduced. The correct amplitude relations are restored by the reciprocal expander in the receiver.

\section{2 $\mu$-Law Companding Profiles}

The $\mu$-Law compander, introduced by Bell Systems, is perhaps the most popular compander in relation to PCM systems and is widely used in North America. The input-output transfer characteristics of a $\mu$-Law compander are described by the formula

$$
y=x_{\text {peak }} \frac{\ln \left(1+\mu \frac{|x|}{x_{\text {peak }}}\right)}{\ln (1+\mu)} \operatorname{sgn}(x)
$$

where $x$ is the instantaneous input signal, $y$ is the companded output signal, $x_{\text {peak }}$ is the maximum input/output signal and sgn is the signum function. The parameter $\mu$ determines the companding profile. The standard value for $\mu$ is 255 and this is normally used with an 8bit converter (e.g. Sklar, 2001). Figure 2 shows the $\mu$-Law compander input-output characteristics for $\mu=0$ (linear) and for $\mu$ varying from 0.1 to 1000 .

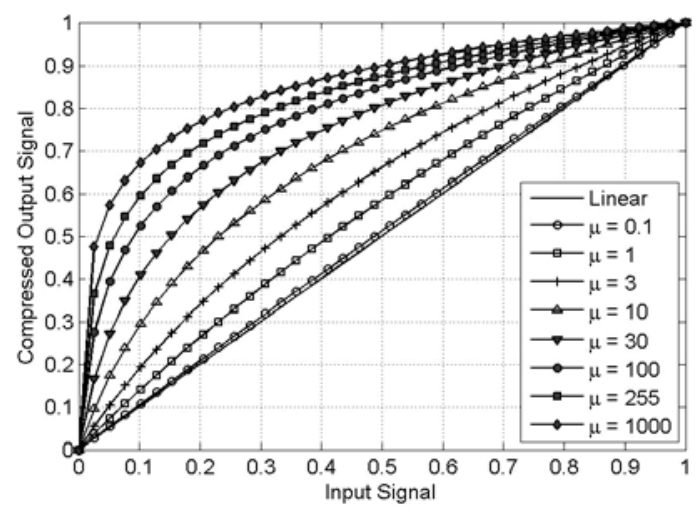

Fig. 2. The $\mu$-Law compander profile for values of $\mu$ from 0 to 1000

\section{3 $\mu$-Law Companding and PAPR Reduction}

From the transfer characteristics of the $\mu$-Law compander, the signals with lower amplitudes are amplified with greater gain than the higher amplitudes signals which are amplified with lower gain. In OFDM systems, the occurrence of subcarriers having very large peak amplitudes is less frequent, while most of the subcarriers have lower peak amplitudes. Because of the less frequent high amplitude subcarriers, the average power is low, resulting in a high PAPR. The high PAPR can be reduced if one of the following is 
possible - either a decrease in the peak amplitudes of the subcarriers or an increase in the average power of the transmitted OFDM signal. If an OFDM signal is passed through a $\mu-$ Law compander which is designed to cover the range of all amplitudes encountered, the subcarrier with the highest peak amplitude will remain relatively unaltered if near the higher levels of the transfer characteristic, while all other subcarriers with lower peak amplitudes will be amplified with varying but larger gains. Thus, the peak power remains relatively unaltered while the average power of the signal is increased due to the companding process. As a result, there is potential reduction in PAPR.

The mathematical expression for the PAPR of general OFDM was given in equation (7). The PAPR for companded OFDM can now be derived. Companding the subcarriers using a $\mu-$ Law profile outlined through equation (10), then it can be shown that the PAPR formula for companded OFDM is given by

$$
P A P R_{\text {comp }}=\max \left(\frac{N \ln \left(1+\mu\left[P_{\text {peak }}\right]^{0.5}\right)}{\sum_{n=1}^{N} \ln \left(1+\mu\left[P_{n}(t)\right]^{0.5}\right)}\right)
$$

where $P_{n}(t)$ is the normalised instantaneous power, i.e. $\left(|x| / x_{\text {peak }}\right)^{2}$, of the $n^{\text {th }}$ subcarrier and $P_{\text {peak }}$ is the normalised peak power from the compander. The maximum PAPR for the companded OFDM transmission occurs for the situation when the data on each subcarrier is the same. In such a situation, the IFFT of the data results in a peak power at one IFFT point and zero power at all other points, similar to the situation outlined in Figure 1 for OFDM with 16 subcarriers carrying the same data and employing QPSK. Thus, the instantaneous power of the first subcarrier, $P_{1}(t)$ will be the same as the peak envelope power, $P_{\text {peak, }}$ and the power at all other subcarriers will be zero. Therefore, the maximum PAPR is given by

$$
P A P R_{\text {comp_max }}=\max \left(\frac{N \ln \left(1+\mu\left[P_{\text {peak }}\right]^{0.5}\right)}{\ln \left(1+\mu\left[P_{\text {peak }}\right]^{0.5}\right)}\right)=N
$$

In this situation, the PAPR of both general OFDM and companded OFDM signals is identical, indicating that companding does not reduce PAPR for this situation. However, when the input data is random, which is presumed to be the normal situation for most applications, then equation (12) indicates that the companding process may be very effective in reducing PAPR. Equation (12) also helps in validating the formulation of PAPR of companded OFDM by showing that the maximum PAPR is the same as the maximum theoretical value of OFDM given by $N$ or $10 \log _{10}(N)$ in $\mathrm{dB}$.

Even though the average and peak envelope power for MPSK and MQAM signal transmissions will be different, it may be shown that the PAPR distributions are in fact identical for all MPSK and MQAM OFDM modulations (e.g. Vallavaraj, 2008). The amplitude levels of MQAM are different from MPSK, which will increase the peak envelope power. However, the average power will also increase in MQAM, thereby making MQAM no different from MPSK as far as the PAPR is concerned. This can be established easily through analytical or simulation studies.

To demonstrate the PAPR reduction arising from companding, QPSK OFDM transmissions can be numerically computed and compared for random data transmission for general 
OFDM and $\mu$-Law companded OFDM with $\mu=255$. Figure 3 shows the conceptual block diagram of the basic Matlab/Simulink simulation model. Random binary sequence data is mapped onto subcarriers using QPSK and then oversampling by a factor of 8 is performed by padding zeros to the baseband modulated signals. The OFDM signals are then obtained by taking the IFFT, from which the instantaneous peak power and the average power over each OFDM symbol can be calculated in order to evaluate the instantaneous PAPR of each OFDM symbol. Simulated results can then be compared with analytical calculations derived from equations (7) and (11).

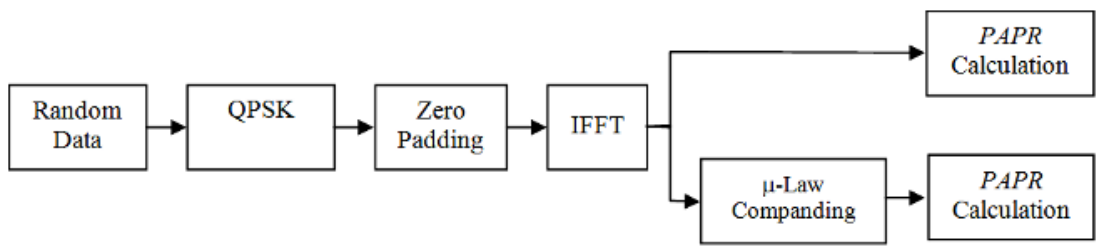

Fig. 3. Block diagram of the basic OFDM model used for the PAPR measurement

Figure 4 displays the maximum PAPR obtained over 1000 OFDM symbols as a function of the number of subcarriers for both normal OFDM and companded OFDM.

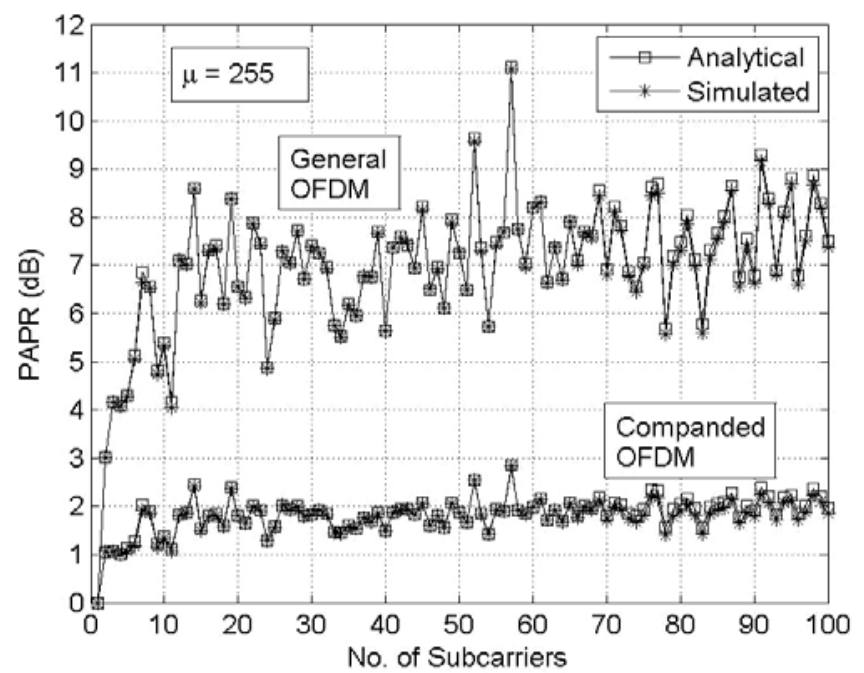

Fig. 4. PAPR as a function of the number of subcarriers for general OFDM and companded OFDM with $\mu=255$

The results show almost identical agreement between all analytical and simulated results. It can also be seen that $\mu$-Law companding with $\mu=255$ provides significant improvement in PAPR over general OFDM transmissions. Specifically, the companding scheme produces a much lower PAPR even for a small number of subcarriers and approaches about $2 \mathrm{~dB}$ as the number of subcarriers increases towards 100 and beyond. 


\section{Companding the WiMax IEEE802.16e DL PUSC}

The investigation into the application of companding specifically to Mobile WiMax with the inclusion of modulated pilots has received only little attention in the literature (Stewart \& Vallavaraj, 2009). To help address this topic the rest of this chapter endeavours to provide a comprehensive simulation investigation of the application of $\mu$-Law companding to one implementation of mobile WiMax where the influence on PSD, BER, PAPR and mobile multipath channel as a function of $\mu$ and modulation is investigated and quantified.

Mobile WiMax uses the principle of OFDMA (OFDM Access) in which a number of subchannels are assigned to different subscribers. The mobile WiMax implementation used in the simulation studies undertaken here is the DL PUSC (Down Link Partially Used SubCarrier). This downlink mode has been selected because it assumes all subchannels can be utilised for information transmission from a base station. Table 1 outlines the parameters of the IEEE802.16e OFDMA configuration employed for the simulation studies. This represents one particular embodiment of the IEEE802.16e standard and is used to demonstrate the application of companding to the system. In the configuration chosen, there are 720 data subcarriers, 184 null subcarriers, 30 subchannels, an IFFT size of 1024, and a guard interval of $1 / 8$ of the 1024 IFFT OFDMA period. The modulations employed are QPSK, 16QAM and 64QAM. Each subchannel comprises 12 subcarriers and 2 pilots.

\begin{tabular}{|c|c|}
\hline Parameter & Value \\
\hline System Bandwidth & $10 \mathrm{MHz}$ \\
\hline IFFT Size & 1024 \\
\hline Modulation & QPSK, 16QAM, 64QAM \\
\hline Subchannels & 30 \\
\hline Null subcarriers & 784 \\
\hline Data subcarriers & 28 subcarriers \\
\hline Cluster size & 14 even symbol + \\
& 14 odd symbol $)$ \\
\hline Clusters per subchannel & 2 \\
\hline Data subcarriers per subchannel & 48 \\
\hline Pilot subcarriers per subchannel & 8 \\
\hline Pilot insertion even symbol & $5^{\text {th }}$ and $9^{\text {th }}$ subcarriers \\
\hline Pilot insertion odd symbol & $1^{\text {st }}$ and $13^{\text {th }}$ subcarriers \\
\hline Pilot values & $4 / 3$ BPSK Modulated \\
\hline Guard Interval $T_{\mathrm{g}}(1 / 8)$ & $12.8 \mu \mathrm{s}$ \\
\hline OFDMA Symbol period $\mathrm{T}_{\mathrm{s}}$ & $115.2 \mu \mathrm{s}$ \\
\hline
\end{tabular}

Table 1. WiMax DL PUSC simulation parameters

Each pilot has a magnitude of $4 / 3$ and is BPSK modulated to conform to the IEEE802.16e standard. The modulation of the pilots uses -1 and +1 PN sequences. Other orthogonal pilot modulation code sequences may be simulated if desired but these can be shown to provide exactly the same results as presented below for the PN sequences. As per the standard, the pilot assignments in the even and odd symbols of clusters in the DL PUSB are shown in Figure 5. These pilots are used for channel estimation and correction. A number of techniques such as piecewise linear interpolation across subcarriers within each cluster, or more advanced techniques such as Least Squares (LS), or Minimum Mean Squares Error 
(MMSE) may be employed (e.g. Hanzo et al., 2003). In the work presented here, when no mobile channel is considered, then the pilots are not utilised for channel estimation or data correction. For simplicity, when a mobile channel is considered in this work a piecewise linear interpolation is applied to correct the channel across the subcarriers independently within the odd and even symbols of each cluster.

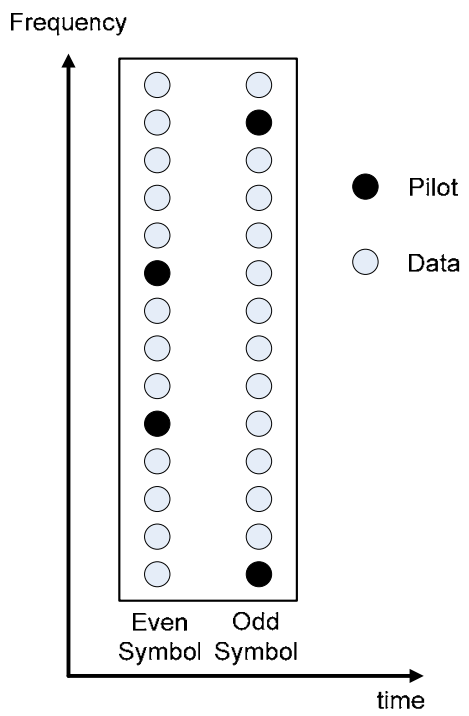

Fig. 5. Pilot assignment for individual OFDMA clusters

In order to set the peak level of the compander in the simulations, 100,000 standard WiMax symbols were transmitted for each of QPSK, 16QAM and 64QAM modulations. The maximum peak amplitude of the set was then chosen as $x_{\text {peak }}$ in the companding equation (10). It may be commented that the peak amplitudes for each of QPSK, 16QAM and 64QAM were within approximately $1.0 \%$ of each other. All modelling and simulation of the WiMax system was again carried out using Matlab/Simulink. To simplify the evaluation of PAPR and BER in the simulation studies of the DL PUSC, the preamble, Reed-Solomon encoding, convolution coding or Turbo coding have not been considered.

To appreciate the general influence of $\mu$-Law companding, Figure 6 compares the relative instantaneous power transmission signals for the same random data over a period of $300 \mu \mathrm{s}$ for 16QAM modulated general WiMax and companded WiMax employing $\mu=30$. In Figure 6 , the instantaneous power is presented in relative terms with respect to the average symbol power of the transmissions. The impact of companding can be immediately appreciated as the PAPR has been significantly reduced. For example, over the $300 \mu$ s time period in Figure 6(a), the peak power for the general WiMax system is approximately 8.2 times larger than the average power producing a PAPR of $9.1 \mathrm{~dB}$. In Figure $6(\mathrm{~b})$ the peak power is approximately 2.6 times larger than the average power for companded WiMax resulting in a PAPR of $4.15 \mathrm{~dB}$. This reduction is a very attractive feature of the companding process. However, the drawback is that when companding is directly applied, the average power of each OFDMA symbol is increased. 


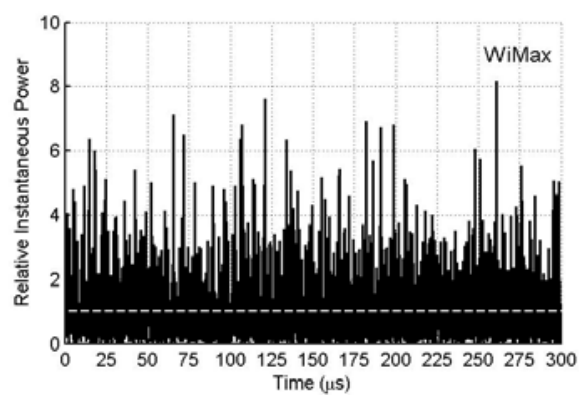

(a) WiMax

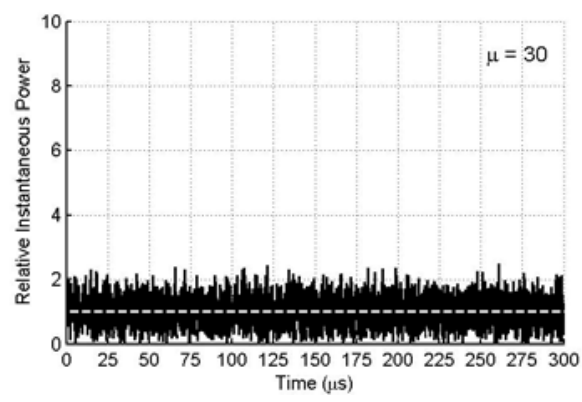

(b) Companded WiMax with $\mu=30$

Fig. 6. Comparison of relative instantaneous power transmissions for (a) standard WiMax, and (b) companded WiMax using $\mu=30$

In order to appreciate this increase in average power, Figure 7 shows the relative average symbol power gain as a function of $\mu$ with respect to the average uncompanded WiMax symbol power for each OFDMA symbol transmission. As the companding profiles are independent of the data modulation employed then it is easy to demonstrate (through simulation) that Figure 7 is also independent of the modulation.

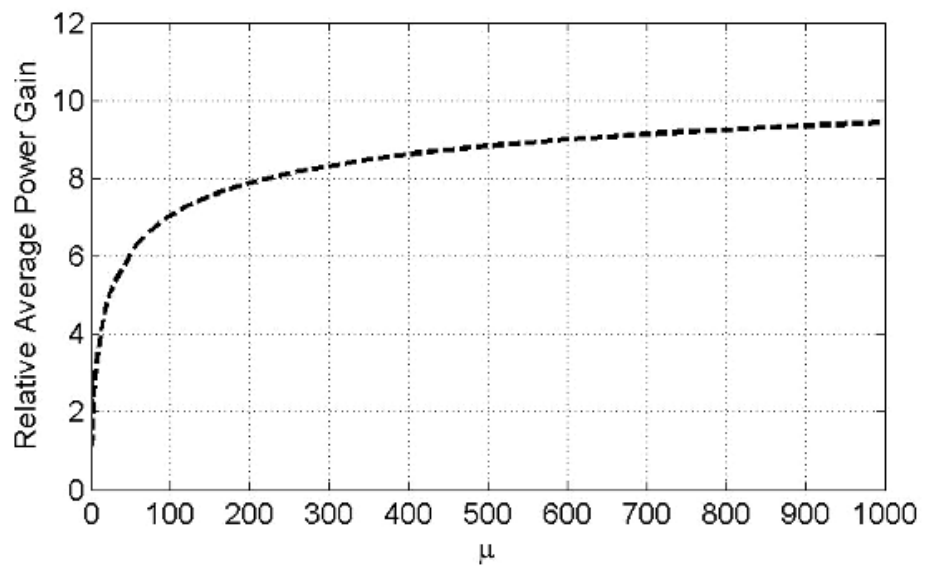

Fig. 7. Relative average power gain of companded WiMax as a function of $\mu$

Clearly as $\mu$ increases from $\mu=0$, i.e. general WiMax, the average power increases rapidly but then becomes asymptotic as $\mu$ tends to higher values. For the PAPR transmission example shown in Figure 6, with $\mu=30$, the average relative power gain is 5.37 times higher than standard WiMax even though the PAPR has dramatically reduced. The issue of increased power due to companding, though recognised, is not often quantified or evaluated in relation to a complete assessment of the significance of companding. The following chapters will attempt to investigate the assessment of both straight companding and also the consideration of companding when the average symbol power of companded transmissions is equalised to the uncompanded WiMax symbol power for each value of $\mu$. 


\section{Spectral Issues Associated With $\mu$-Law Companding}

The averaged PSD of WiMax and companded WiMax as a function of $\mu$ between 0.1 and 255 is shown in Figures 8(a) and (b). These figures compare the PSD for both the direct application of companding and also the equalised power companded situations. The averages were performed over 2000 symbol transmissions to provide accuracy in the results.

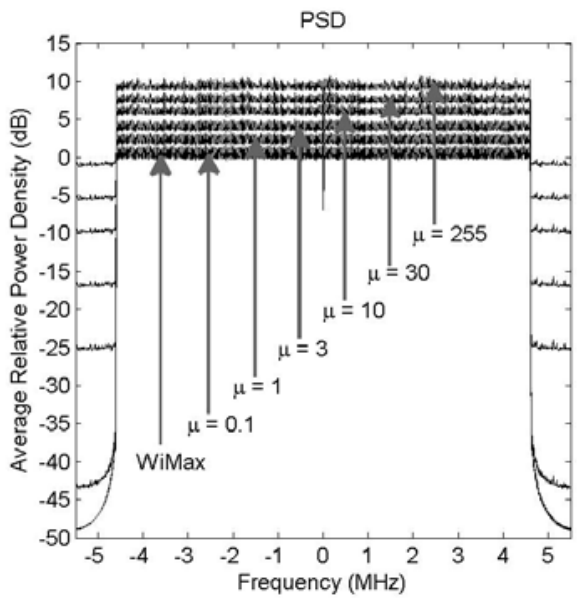

(a) Companded

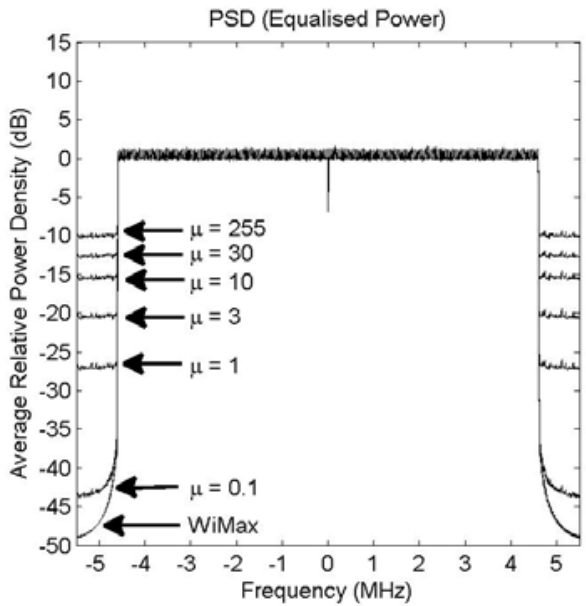

(b) Companded equalised power

Fig. 8. Averaged PSDs of general WiMax and companded WiMax as a function of $\mu$ between 0.1 and 255 for (a) $\mu$-Law companding, (b) $\mu$-Law companding employing equalised averaged symbol power

The presence of increased in-band spectral power as a function of increasing values of $\mu$ is clearly evident in Figure 8(a), where spectral power density re-growth has occurred within the signal bandwidth. This phenomenon is appreciated in the literature and as discussed above is an artefact of the raw companding process. As an example, for $\mu=255$, the in-band PSD level has increased by around $9 \mathrm{~dB}$ from the normal uncompanded WiMax situation. This is in general agreement with the power gain curve in Figure 7 for $\mu=255$. However, out-of-band spectral splatter is also introduced and increases with increasing $\mu$. It can be seen that the rapid or sharp roll-off of the PSD outside the signal bandwidth for $\mu$ values still exists, but the base of the bandwidth skirt rises significantly as $\mu$ increases. For $\mu=255$, it can be seen that the base of the skirt is only around $10 \mathrm{~dB}$ lower than the in-band PSD. In the equalised power situation, it is easier to appreciate that small values of $\mu$ introduce lower levels of out-of-band energy and will therefore potentially cause less interference.

Internal spectral re-growth will assist in improving BER when raw campanding is applied and this may be considered a natural advantage of companding. In reality, if power is to be equalised for all values of $\mu$, then a critical assessment of how BER variations with equalised power must be understood. However, a further important issue in relation to PSD is that the presence of any increased out-of-band spectral power will cause inter channel interference if not addressed. Clearly choice of very small values of $\mu$ will assist in reducing this 
interference. Application of suitable digital filtering may also be employed but this in itself has to be carefully considered as phase variations associated with filter roll-off must be appreciated around the bandwidth edges otherwise inherent BER will occur at the receiver if not properly corrected.

\section{BER Evaluation as a Function of $\mu$}

The BER for companded WiMax and equalised power companded WiMax has been evaluated as a function of $\mu$ over 0.1 to 1000 for QPSK, 16QAM and 64QAM modulations. The results are shown in Figures 10(a) -10(f) (see next page) where the BER probability for each situation is plotted as a function of the SNR. These graphs demonstrate two main aspects related to companding. Firstly, for the straight companding situations (i.e. Figures 10(a), (c) and (e)), as $\mu$ increases from zero (i.e. from standard WiMax), the BER performance starts to improve as a direct consequence of the increased power. However, after a certain value of $\mu$ the BER performance starts to degrade and the curves gradually move outwards towards higher values of SNR.

The reason for this can be understood as follows. As $\mu$ increases from 0 towards larger values, the companding profiles presented in Figure 2 indicate that for larger range input signals there is less range variation on the companded output signals. The signals are of course expanded through decompanding at the receiver. When noise is present for larger $\mu$ values, even a small noise variation on a large amplitude signal can produce significant variations on the decompressed amplitude signal at the receiver thus causing larger bit errors to be produced. To understand the behaviour more accurately, and to determine optimized $\mu$ values which produce best or optimised BER performance for companding it is possible to plot the SNR value which produces a BER of 0.001 as a function of $\mu$ and each modulation. These plots are shown in Figure 9.

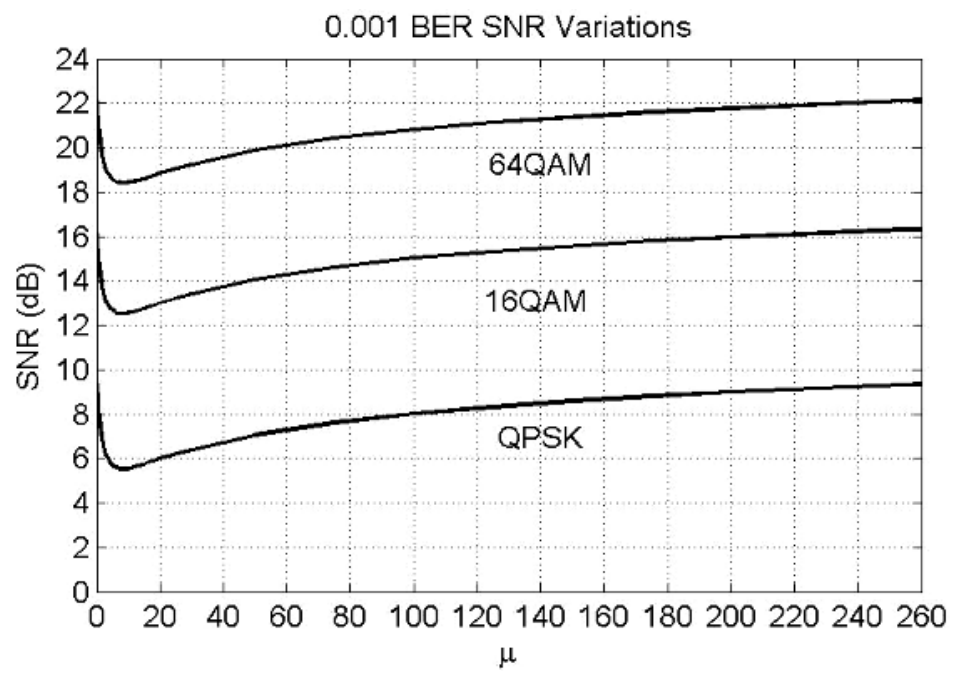

Fig. 9. The variation in SNR as a function of $\mu$ and modulation for companded WiMax for a BER probability of 0.001 


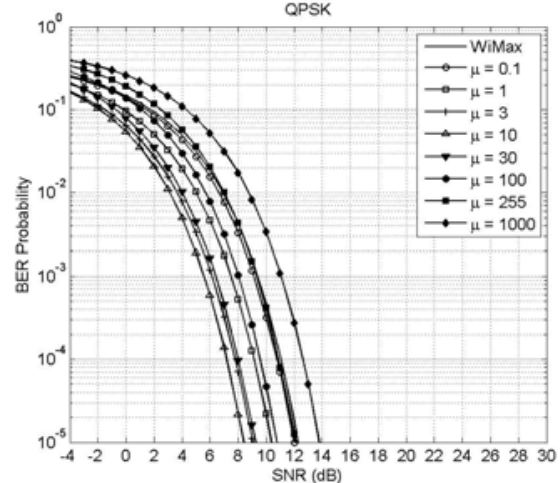

(a) Companded QPSK

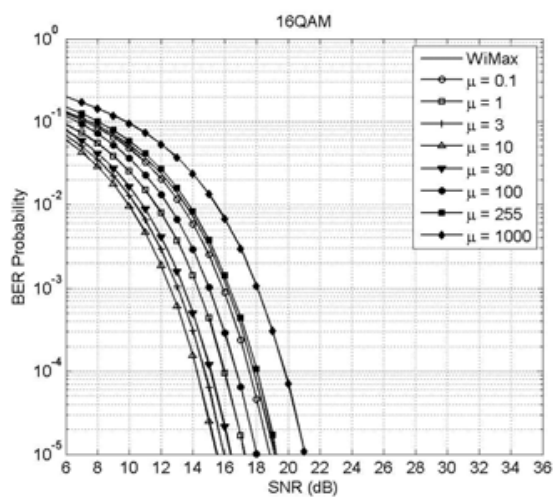

(c) Companded 16QAM

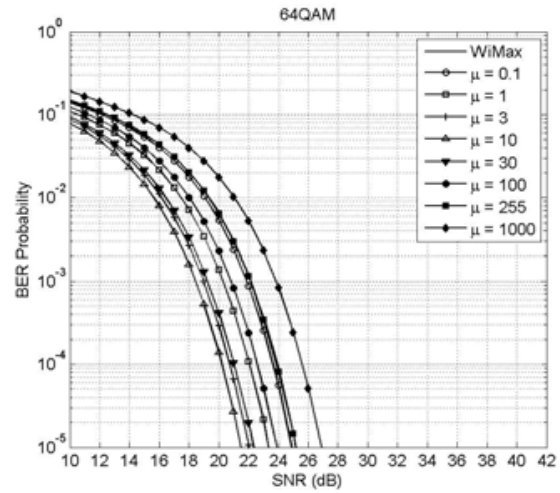

(e) Companded 64QAM

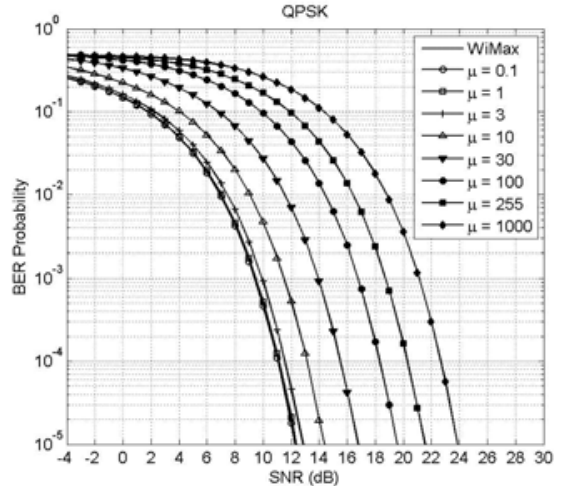

(b) Companded QPSK Equalised Power

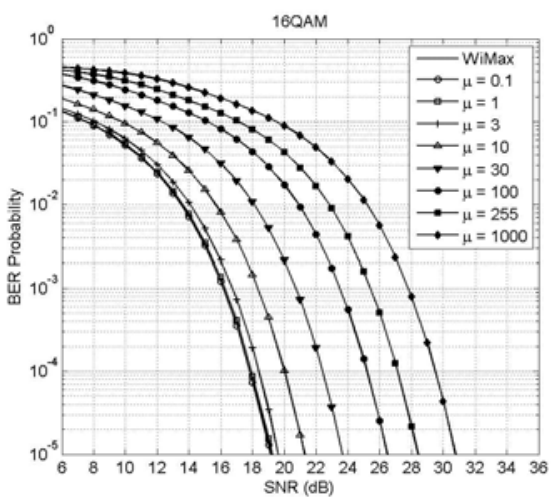

(d) Companded 16QAM Equalised Power

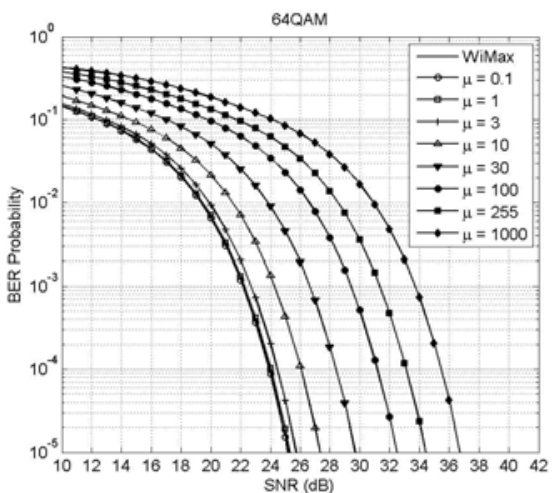

(f) Companded 64QAM Equalised Power

Fig. 10. Evaluation of BER probabilities against SNR as a function of $\mu$ for companded WiMax and also companded WiMax with equalised symbol power - QPSK BER curves are shown in (a) and (b), 16QAM in (c) and (d), and 64QAM in (e) and (f) 
Figure 9 demonstrates that the BER improves quickly (due to the decrease in SNR) for low values of $\mu$ but then gradually degrades as the companding profile changes for larger $\mu$ values. The change in SNR is also seen to be similar for each modulation. The optimised $\mu$ value which achieves best BER performance (i.e. achieves the best SNR) for each modulation is shown in Table 2 .

\begin{tabular}{|c|c|}
\hline Modulation & Optimised $\mu$ \\
\hline QPSK & $\mu=8$ \\
\hline 16QAM & $\mu=8$ \\
\hline 64QAM & $\mu=8$ \\
\hline
\end{tabular}

Table 2. Optimised BER $\mu$ values for companded WiMax

Table 2 demonstrates that the optimized $\mu$ value is independent of the modulation used in WiMax. From Figure 9 there is also very little variation in BER around the optimized $\mu$ values i.e. around $\mu=7$ to 9. The BER curves for QPSK, 16QAM and 64QAM modulations for optimised $\mu=8$ are displayed in Figure 11 alongside the uncompanded WiMax BER curves. These curves allow the optimized BER performance of companded WiMax to be made with standard WiMax.

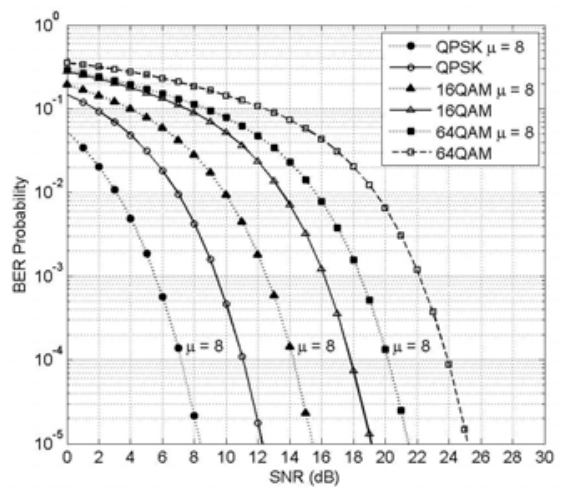

Fig. 11. BER probability comparison between optimised companding with $\mu=8$, and WiMax as a function of the modulation

However, the BER graphs of Figures 10 (a), (c) and (e) may be misleading as any WiMax transmitter must still operate under the constraints of actual real output power requirements. In this situation, the equalised power BER graphs represented in Figure 10 (b), (d) and (f) should be consulted. These curves indicate that when equalised symbol power is considered for each value of $\mu$, then as $\mu$ is increased the BER actually degrades for all SNR situations. This indicates, as expected, that in terms of output power requirements there is in reality a reduction in BER arising as an artefact of the companding process. However, it should be noted that the degradation in BER is not too significant for reduced values of $\mu$ indicating that for companding curves associated with lower values of $\mu$, the decrease in BER performance is not severe. Simulations demonstrate that the cost in terms of increased SNR when companding is applied is nearly independent of whether QPSK, 
16QAM or 64QAM modulation is utilised. The difference in increased SNR cost across all simulations was in agreement to approximately $\pm 0.1 \mathrm{~dB}$ for each value of $\mu$. This is expected as the increased SNR companding power increase also appears to be independent of the modulation from the optimised results shown in Figure 9 and discussed above. The cost in terms of increased SNR, denoted by $\Delta$ SNR, as a function of $\mu$ for a BER probability of 0.001 is plotted in Figure 12(a) for the range of $\mu=0$ to 50 to show how variations in $\Delta$ SNR occur for smaller $\mu$ values, and also in Figure 12(b) for the general range between $\mu=0$ to 1000 .

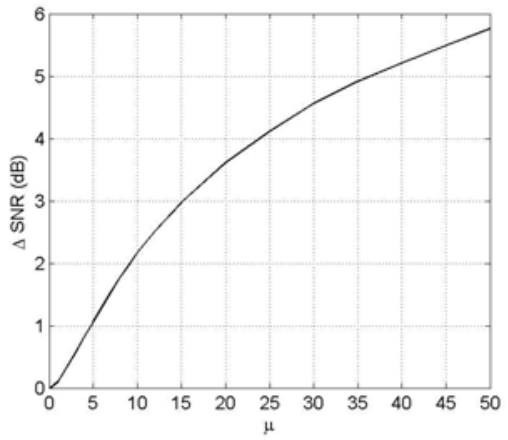

(a) $\mu=0$ to 50

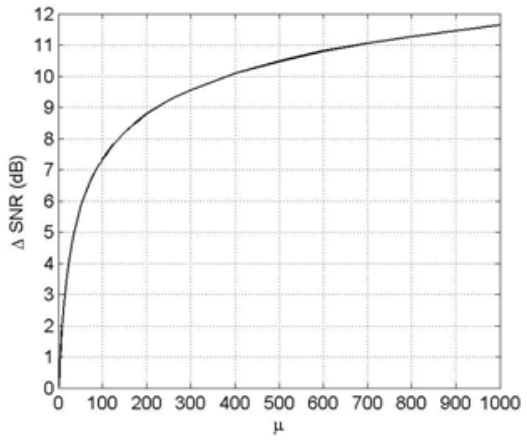

(b) $\mu=0$ to 1000

Fig. 12. The variations in $\Delta$ SNR as a function of $\mu$ for (a) $\mu$ values in the range 0 to 50 , and (b) $\mu$ values in the general range 0 to 1000

Figures 12(a) and (b) are important results as they allow a designer to choose a suitable companding profile knowing the BER degradation or reduction in SNR expected as a function of $\mu$. Ultimately this degradation in BER performance can be chosen in conjunction with the desired PAPR required when companding is considered. Quantification of the reduction in PAPR as a function of $\mu$ will now be presented in the next section.

\section{PAPR Reduction as a Function of $\mu$}

It has been shown previously that the PAPR is independent of the modulation employed in the data transmissions of OFDM systems. This is also easily verified for the WiMax simulations as well. Through simulation, the PAPR values of 100,000 DL PUSB WiMax symbols were evaluated for QPSK, 16QAM and 64QAM. The conventional PAPR Complementary Cumulative Distribution Function (CCDF) distributions were then constructed. The CCDFs represent the PAPR probability distributions and allow the probability of a PAPR value of any WiMax symbol exceeding a certain value (denoted by $\mathrm{PAPR}_{0}$ ) to be determined. In agreement with previous general investigations of companded OFDM systems, the PAPR CCDF distributions of both non-companded and companded OFDM signals is dependent only on the number of subcarriers and is independent of the type of modulation used (e.g. Vallavaraj, 2008; Stewart and Vallavaraj, 2008). For the CCDFs in each of the modulation situations for WiMax, the same values within PAPR tolerances of approximately $\pm 0.1 \mathrm{~dB}$ were obtained at the 0.001 probability level. The PAPR CCDFs as a function of $\mu$ are shown in Figure 13. Also shown in Figure 13 is the CCDF curve for the 
optimised non-equalised power value of $\mu=8$. It can be seen that as $\mu$ increases, the PAPR can be significantly reduced. Though it is attractive to reduce the PAPR to very low levels, the disadvantage of companding is the inherent compromise in BER for equalised power situations and spectral splatter as outlined in the previous sections. However, it is worthy to note that for small values of $\mu$, the PAPR reduction can still be significant without severe compromise to the BER. For example, even with $\mu=1$, at the 0.001 probability level the PAPR reduces from around $11.7 \mathrm{~dB}$ to $9.9 \mathrm{~dB}$. For the optimized non-equalised power situation, $\mu=8$, the PAPR is reduced from around $11.7 \mathrm{~dB}$ to $6.6 \mathrm{~dB}$, a reduction of $5.1 \mathrm{~dB}$.

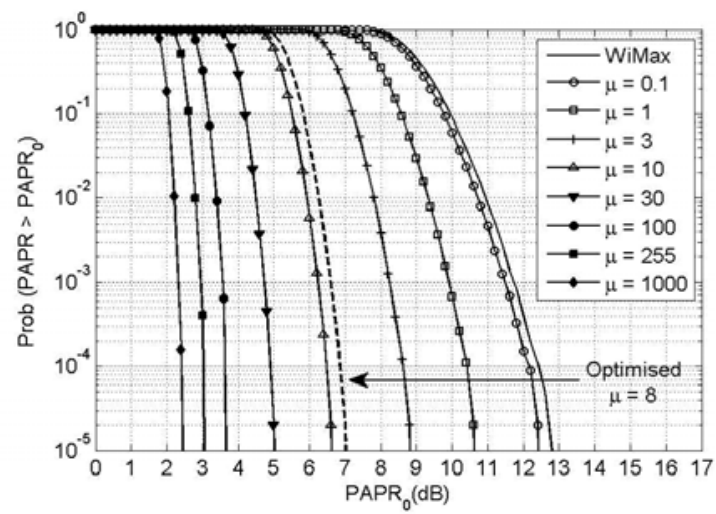

Fig. 13. Companded WiMax CCDF curves as a function of $\mu$

Figure 14 quantifies the PAPR reduction at the CCDF 0.001 probability level as a function of $\mu$. Two ranges of $\mu$ are presented to allow a more detailed appreciation of the PAPR levels achieved for lower values of $\mu$ as well as the general reduction in PAPR up to $\mu=1000$.

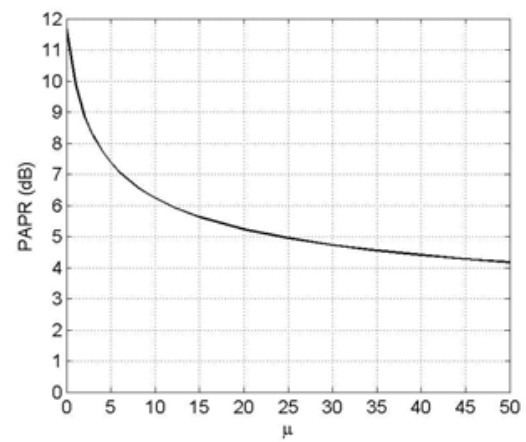

(a) $\mu=0$ to 50

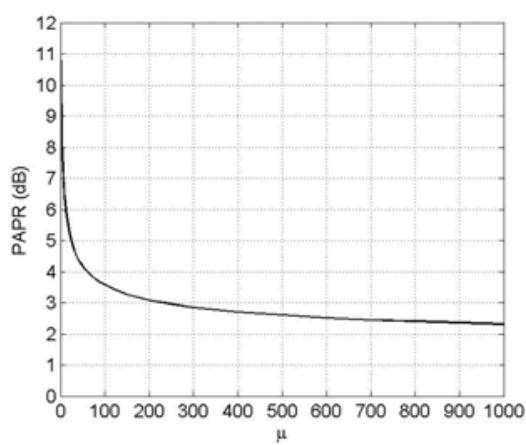

(b) $\mu=0$ to 1000

Fig. 14. WiMax PAPR levels at the CCDF 0.001 probability level for (a) the range $\mu=0$ to 50 and (b) the general range $\mu=0$ to 1000

For situations of improved PAPR a compromise is required to be struck between and desired PAPR and any acceptable degradation in BER. The BER and CCDF figures 
presented in this chapter will therefore allow decisions to be made between PAPR reduction and expected BER levels for specific application requirements.

\section{The Influence of Mobility on Companding}

The influence of companding on the BER performance of OFDM systems when mobility is present has also received little general attention in the literature. The effect of companding within mobile environments for WiMax is investigated here for two specific standard channel models, viz. the WiMax ITU fixed Vehicular A (Veh A) channel and the WiMax ITU mobile Pedestrian B (Ped B) channel. Veh A represents mobility in a vehicular environment while Ped B represents pedestrian mobility. The specific relative multipath delays and average powers associated with these channels are outlined below in Table 3 .

\begin{tabular}{|c|c|c|c|}
\multicolumn{2}{|c|}{ Veh A } & \multicolumn{2}{c|}{ Ped B } \\
\hline Delay (ns) & Power (dB) & Delay (ns) & Power (dB) \\
\hline 0 & 0 & 0 & -3.9 \\
310 & -1 & 200 & -4.8 \\
710 & -9 & 800 & -8.8 \\
1090 & -10 & 1200 & -11.9 \\
1730 & -15 & 2300 & -11.7 \\
2510 & -20 & 3700 & -27.8 \\
\hline
\end{tabular}

Table 3. Channel parameters for the ITU Veh A and Ped B simulations

The channel correction method employed in this study is a simple piecewise linear interpolation between pilots in each individual symbol within each cluster. More advanced channel correction methods have not been considered in this study as the purpose is to establish the general influence of mobility on companded WiMax.

Speeds of $60 \mathrm{kmh}^{-1}$ for Veh A and $3 \mathrm{kmh}^{-1}$ for Ped B have been considered for evaluation of the influence of companding on the BER performance. Assuming a carrier frequency of approximately $2.4 \mathrm{GHz}$, then the appropriate maximum Doppler frequencies related to mobility can be incorporated in the Matlab/Simulink channel models. Table 4 shows the adopted mobile speeds and the associated Doppler frequencies used for the simulations models.

\begin{tabular}{|c|c|c|c|}
\hline \multicolumn{2}{|c|}{ Veh A } & \multicolumn{2}{c|}{ Ped B } \\
\hline Speed & Doppler Frequency & Speed & Doppler Frequency \\
\hline $60 \mathrm{kmh}^{-1}$ & $133.33 \mathrm{~Hz}$ & $3 \mathrm{kmh}^{-1}$ & $6.67 \mathrm{~Hz}$ \\
\hline
\end{tabular}

Table 4. Speeds and associated maximum Doppler frequencies used in the WiMax channel simulation models for Veh A and Ped B channels

Figures 15(a)-(f) display the results for mobility at $60 \mathrm{kmh}^{-1}$ in the Veh A channel as a function of QPSK, 16QAM and 64QAM modulation for general WiMax, companded WiMax and equalised power companded WiMax over $\mu=0.1$ to 1000. Figures. 16(a)-(f) display the results for mobility at $3 \mathrm{kmh}^{-1}$ in the Ped B channel for the same parameters evaluated for Figure 15. 


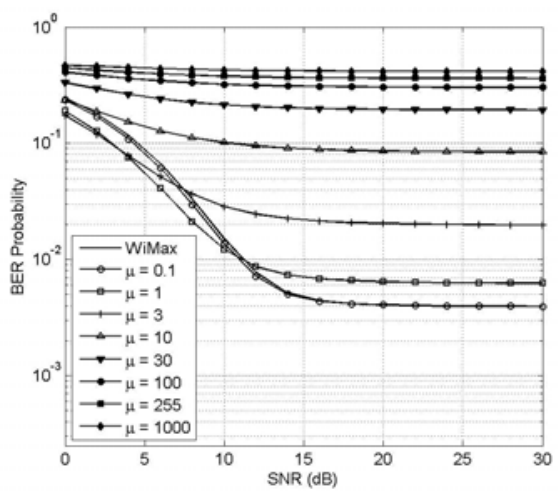

(a) QPSK Veh A

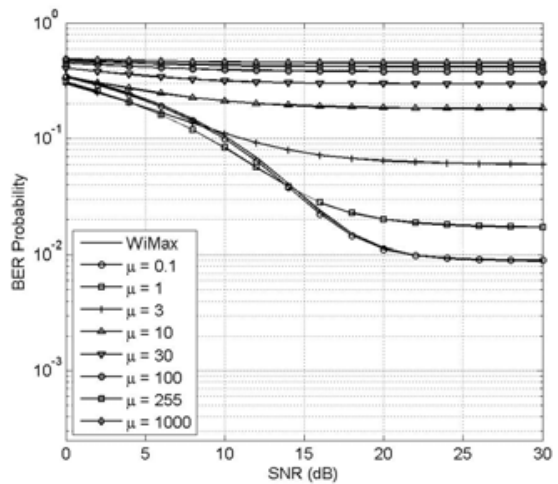

(c) 16QAM Veh A

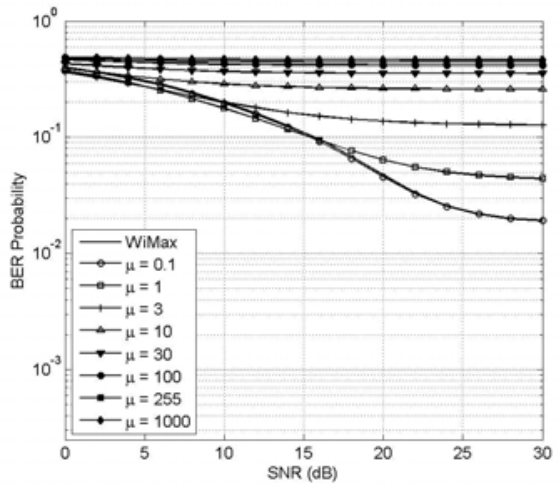

(e) 64QAM Veh A

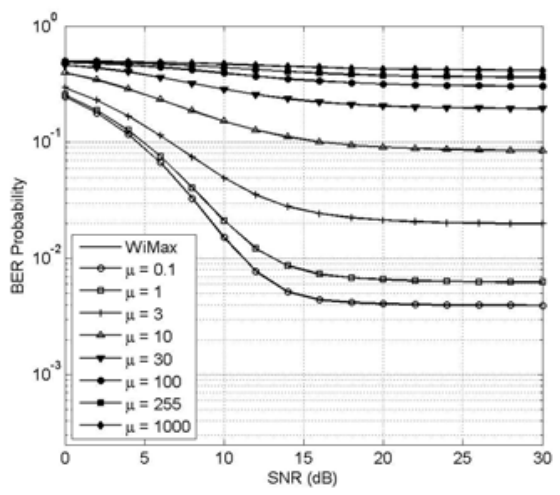

(b) QPSK Veh A Equalised Power

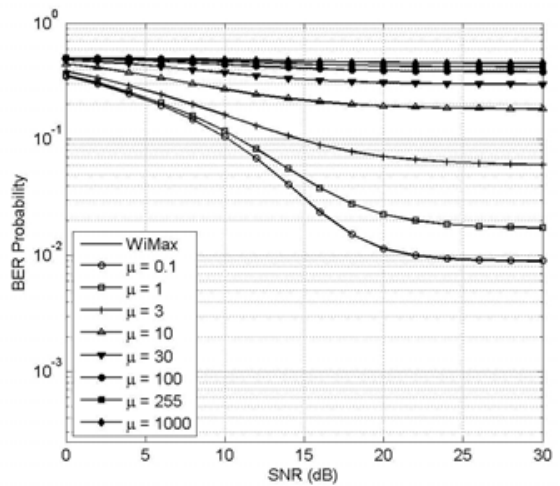

(d) 16QAM Veh A Equalised Power

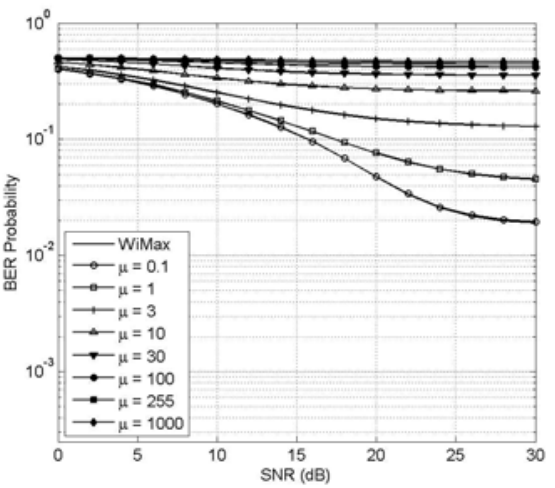

(f) 64QAM Veh A Equalised Power

Fig. 15. QPSK, 16QAM and 64QAM Veh A BER probability curves as a function of $\mu$ for situations of companded and equalised power companded WiMax 


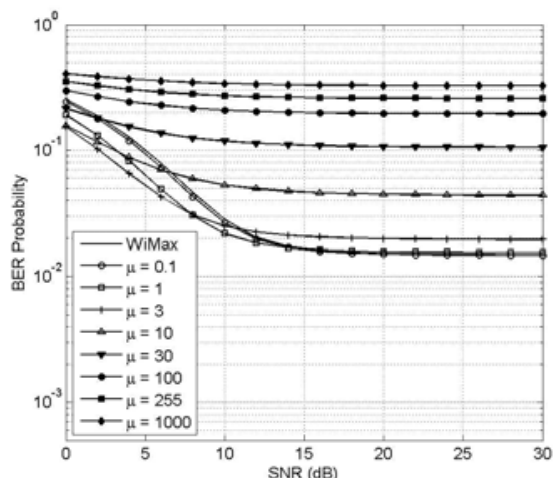

(a) QPSK Ped B

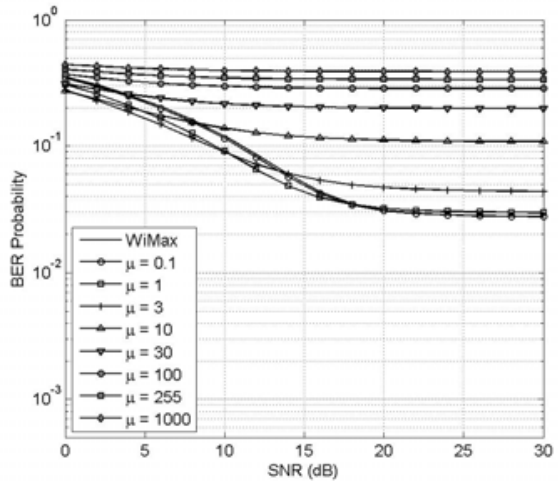

(c) 16QAM Ped B

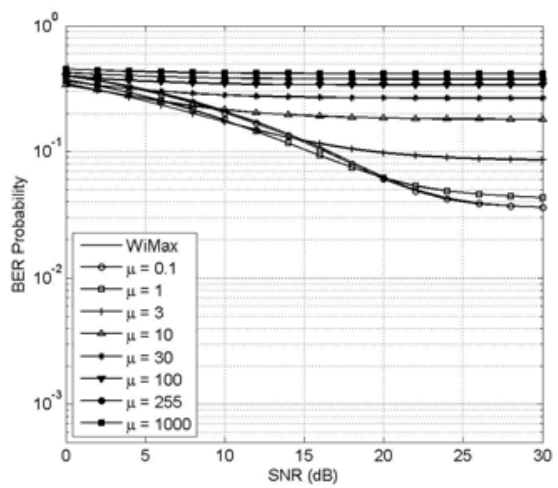

(e) 64QAM Ped B

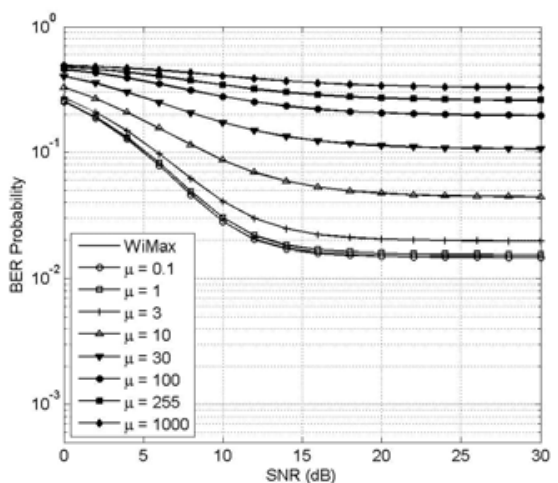

(b) QPSK Ped B Equalised Power

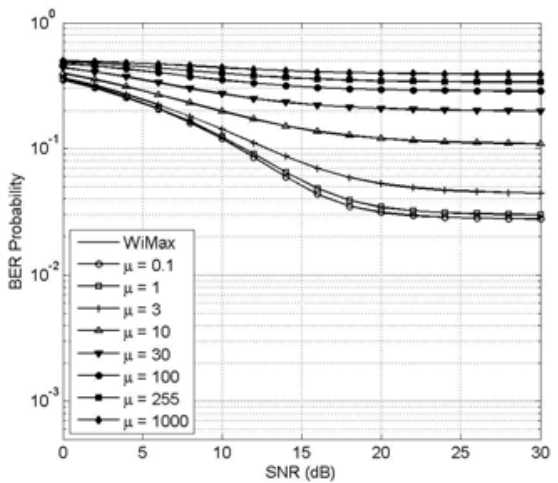

(d) 16QAM Ped B Equalised Power

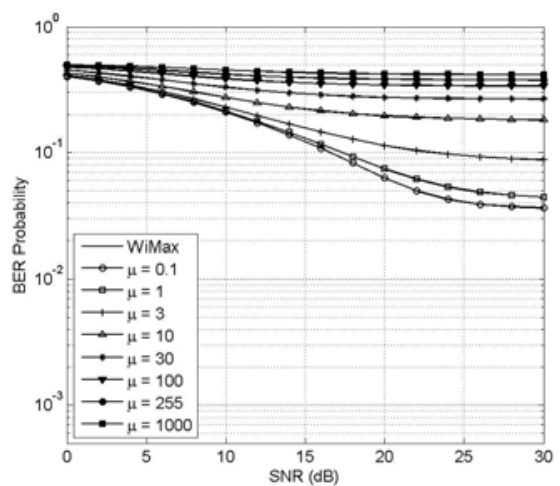

(f) 64QAM Ped B Equalised Power

Fig. 16. QPSK, 16QAM and 64QAM Ped B BER probability curves as a function of $\mu$ for situations of companded and equalised power companded WiMax 
The significant observations regarding mobility are as follows. The BER depreciates significantly for Veh A and Ped B channels as $\mu$ increases for both companded and equalised power companded situations. It can also be seen that for each value of $\mu$, as the SNR increases, the BER flattens off to an asymptotic optimum value. This asymptotic value decreases with increasing $\mu$, and also with increased data modulation on the subcarriers i.e. the performance is best for QPSK, deteriorates for 16QAM and further deteriorates for 64QAM. Thus a general conclusion is that increased companding will always degrade the performance of WiMax systems for larger SNR in the mobile channels considered. It may also be noted that for very small values of $\mu$, the BER performance in the asymptotic region is comparable to the asymptotic value associated with standard WiMax. The main reason for the depreciated BER performance is clearly a combination of the companding profile, the modulation and also the affects of the channel.

Interestingly, for the direct companding situations, there is a marginal improvement in BER rate over WiMax at lower SNR values across a range of $\mu$ values. An improvement in BER with companding is expected due to the inherent increased average power provided through the companding process itself. However, the BER is still poor over the regions where the improvement over WiMax occurs. For the Veh A scenarios in Figure 15(a), (b) and (c), the value of $\mu$ which optimises the BER varies over the lower SNR range under consideration. The optimised $\mu$ values over the lower SNR range is also nearly independent of the modulation employed. For example, for QPSK, 16QAM and 64QAM, for SNR $<4 \mathrm{~dB}$ the curve for $\mu \approx 3$ provides the best BER performance. For the approximate range $4 \mathrm{~dB}<$ SNR $<11 \mathrm{~dB}$, the curve for $\mu \approx 1$ is best, and for $11 \mathrm{~dB}<\mathrm{SNR}<16 \mathrm{~dB}, \mu \approx 0.1$ is optimum. Above $16 \mathrm{~dB}$ WiMax provides the best BER performance, although there is minimal difference for values of $\mu$ around 1 or less than 1 and WiMax as the BER levels off.

For the Ped B channel in Figures 16(a), (c) and (e), the best performance of companding for lower values of SNR appears to be more dependent on the modulation. For the QPSK BER curves evaluated, for $\mathrm{SNR}<8 \mathrm{~dB}, \mu \approx 3$ is preferred, for $\mathrm{SNR}>8, \mu \leq 1$ is best, though values of $\mu$ around 1 provide similar results to WiMax in this situation. For 16QAM, $\mu \approx 3$ is preferred for SNR $<10 \mathrm{~dB}$, and for $10 \mathrm{~dB}<\mathrm{SNR}>18 \mathrm{~dB} \mu \approx 1$ is preferred and for SNR $>$ $18 \mathrm{~dB} \mu \approx 0.1$ is best. For $64 \mathrm{QAM}, \mu \approx 3$ is preferred for $\mathrm{SNR}<11 \mathrm{~dB}$, whilst for the range $11 \mathrm{~dB}$ $<\mathrm{SNR}<20 \mathrm{~dB} \mu \approx 1$ produces the best BER, and for SNR $>20 \mathrm{~dB}, \mu \leq 0.1$ is the best. Again for increasing SNR WiMax produces the best asymptotic BER performance, though there is little difference in BER performance between WiMax and very small values of $\mu$ as the BER levels off. Clearly, the BER performance in lower SNR values, when mobility is present, depends not just on the companding profile, but on the modulation and the nature of the multipath channel.

As discussed previously, the raw companding BER curves may be slightly misleading due to the fact that real transmitters may be required to work on power limitations in which case the equalised symbol power curves are important. For the equalised power situations, as expected, as $\mu$ increases, the BER performance depreciates. However, for very small values of $\mu$, in all situations, the companded performance is similar or close to the general WiMax situation. The reason for the rapid deterioration in BER with increasing $\mu$ can be explained again as a consequence of the nature of the companding profiles, i.e. large peak amplitude signals can have significant decompanding bit errors at a receiver for larger $\mu$ values when noise is present. This, mixed with the problems of a mobile channel, accentuates the deterioration in BER. However, for some situations the increased BER may be acceptable 
within some mobile channels when a significant improvement in PAPR is desired. But perhaps the most important result is that the asymptotic BER values for the equalised power companded situations are nearly identical to the raw companded asymptotic BER values. These asymptotic values are plotted in Figure 17 and indicate that for large SNR values, when $\mu$ is applied, the influence of the multipath channel is the overwhelming limiting factor on the BER performance. Figure 17 is therefore useful to precisely quantify the optimum BERs achievable for the Veh A and Ped B channels when companding is applied.

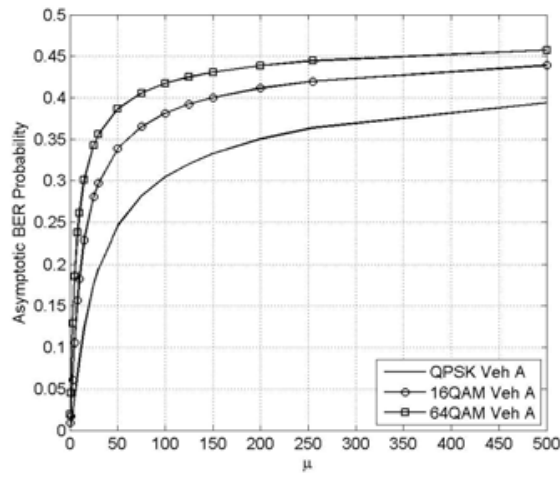

(a) Veh A $60 \mathrm{kmh}^{-1}$

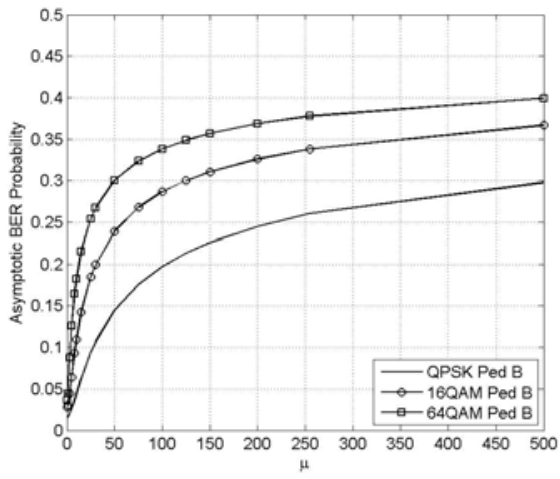

(b) Ped B 3kmh-1

Fig 17. Variation of the asymptotic BER values as a function of $\mu$ for QPSK, 16QAM and 64QAM for (a) Veh A $60 \mathrm{~km}^{-1}$ and (b) Ped B $3 \mathrm{kmh}^{-1}$

\section{Conclusions}

This chapter has presented and discussed the principles of PAPR reduction and the principles of $\mu$-Law companding. The application of $\mu$-Law compounding was applied to one implementation of mobile WiMax using an FFT/IFFT of 1024. The main conclusions are as follows. Companding using $\mu$-Law profiles has the potential to reduce significantly the PAPR of WiMax. For straight companded WiMax the average power increases and as a consequence the BER performance can be improved. For direct companding the optimum BER performance occurs for $\mu=8$, which produces a PAPR of approximately $6.6 \mathrm{~dB}$ at the 0.001 probability level, i.e. a reduction of $5.1 \mathrm{~dB}$. However an increase in frequency spectral energy splatter occurs which must be addressed to minimise inter channel interference. For equalised symbol power companded transmissions, the BER performance is actually shown to deteriorate for all values of $\mu$. However, for small values of $\mu$, the BER degradation is not severe. This is advantageous as a balance between cost in terms of BER and PAPR reduction can now be quantified along with the expected out-of-band PSD for any chosen value of $\mu$. The figures produced in this chapter will allow an engineer to take informed decisions on these issues. In relation to mobility, the influence of companding on performance is more complex and appears to depend on the modulation, mobile speed and more importantly the nature of the channel itself. It was shown that for straight companding the optimum BER performance at low values of SNR was dependent on the value of $\mu$ as well as the nature of the channel. Different ranges of lower SNR values defined different optimum values of $\mu$. 
Generally, for larger SNR values the BER performance degraded as $\mu$ was increased and became asymptotic with increasing SNR. For the equalised power companding situation, WiMax always produces best BER performance. However, for very small values of $\mu$, there is very little difference between companded WiMax and WiMax. A compromise may also be reached in relation to a reduced BER performance in mobility versus a required PAPR level. It was also discovered that the companded and equalised power companded BER optimised asymptotic values for mobility were approximately the same indicating that the best BER performance for the minimum SNR requirements can be quantified for any design value of $\mu$. This is also helpful in understanding the anticipated best BER performance available in mobile channels when companding is chosen to provide a reduced PAPR level.

Further work in relation to the results presented in this chapter may be carried out. This includes an investigation of the BER performance for companded WiMax when channel coding is incorporated, i.e. convolution and turbo coding, when Reed-Solomon coding is employed, and when other more advanced channel estimation techniques are considered. The importance of filtering or innovative techniques for reducing the spectral splatter should also be explored. Other areas for investigation also include quantifying the influence on BER for a larger range of different mobile channels as a function of $\mu$.

\section{References}

Armstrong, J. (2001). New OFDM Peak-to-Average Power Reduction Scheme, Proc. IEEE, VTC2001 Spring, Rhodes, Greece, pp. 756-760

Armstrong, J. (2002). Peak-to-average power reduction for OFDM by repeated clipping and frequency domain filtering, Electronics Letters, Vol.38, No.5, pp.246-247, Feb. 2002.

Bäuml, R.W.; Fisher, R.F.H. \& Huber, J.B. (1996). Reducing the peak-to-average power ratio of multicarrier modulation by selected mapping, IEE Electronics Letters, Vol.32, No.22, pp. 2056-2057

Boyd, S. (1986). Multitone Signal with Low Crest Factor, IEEE Transactions on Circuits and Systems, Vol. CAS-33, No.10, pp. 1018-1022

Breiling, M.; Müller-Weinfurtner, S.H. \& Huber, J.B. (2001). SLM Peak-Power Reduction Without Explicit Side Information, IEEE Communications Letters, Vol.5, No.6, pp. 239241

Cimini, L.J.Jr.; \& Sollenberger, N.R. (2000). Peak-to-Average Power Ratio Reduction of an OFDM Signal Using Partial Transmit Sequences, IEEE Communications Letters, Vol.4, No.3, pp. 86-88

Davis, J.A. \& Jedwab, J. (1999). Peak-to-Mean Power Control in OFDM, Golay Complementary Sequences, and Reed-Muller Codes, IEEE Transactions on Information Theory, Vol. 45, No.7, pp. 2397-2417

De Wild, A. (1997). The Peak-to-Average Power Ratio of OFDM, MSc Thesis, Delft University of Technology, Delft, The Netherlands, 1997

Golay, M. (1961). Complementary Series, IEEE Transactions on Information Theory, Vol.7, No.2, pp. 82-87

Hanzo, L.; Münster, M; Choi, B.J. \& Keller, T. (2003). OFDM and MC-CDMA for Broadcasting Multi-User Communications, WLANS and Broadcasting, Wiley-IEEE Press, ISBN 0470858796 
Han, S.H. \& Lee, J.H. (2005). An Overview of Peak-to-Average Power Ratio Reduction Techniques for Multicarrier Transmission, IEEE Wireless Communications, Vol.12, Issue 2, pp. 56-65, April 2005

Hill, G.R.; Faulkner, M. \& Singh, J. (2000). Reducing the peak-to-average power ratio in OFDM by cyclically shifting partial transmit sequences, IEE Electronics Letters, Vol.33, No.6, pp. 560-561

Huang, X.; Lu, J., Chang, J. \& Zheng, J. (2001). Companding Transform for the Reduction of Peakto-Average Power Ratio of OFDM Signals, Proc. IEEE Vehicular Technology Conference 2001, pp. 835-839

IEEE Std. 802.16e. (2005). Air Interface for Fixed and Mobile Broadband Wireless Access Systems: Amendment for Physical and Medium Access Control Layers for Combined Fixed and Mobile Operation in Licensed Bands, IEEE, New York, 2005.

Jayalath, A.D.S. \& Tellambura, C. (2000). Reducing the Peak-to-Average Power Ratio of Orthogonal Frequency Division Multiplexing Signal Through Bit or Symbol Interleaving, IEE Electronics Letters, Vol.36, No.13, pp. 1161-1163

Jiang, T. \& Song, Y-H. (2005). Exponential Companding Technique for PAPR Reduction in OFDM Systems, IEEE Trans. Broadcasting, Vol. 51(2), pp. 244-248

Jones, A.E, \& Wilkinson, T.A. (1995). Minimization of the Peak to Mean Envelope Power Ratio in Multicarrier Transmission Schemes by Block Coding, Proc. IEEE VTC'95, Chicago, pp. 825-831

Jones, A.E. \& Wilkinson, T.A (1996). Combined Coding for Error Control and Increased Robustness to System Nonlinearities in OFDM, Proc. IEEE VTC'96, Atlanta, GA, pp. 904-908

Jones, A.E.; Wilkinson, T.A. \& Barton, S.K. (1994). Block coding scheme for the reduction of peak to mean envelope power ratio of multicarrier transmission schemes, Electronics Letters, Vol.30, No.25, pp. 2098-2099

Kang, S.G. (2006). The Minimum PAPR Code for OFDM Systems, ETRI Journal, Vol.28, No.2, pp. 235-238

Lathi, B.P. (1998). Modern Digital and Analog Communication Systems, 3rd Ed., pp. 262-278, Oxford University Press, ISBN 0195110099

Li, X. \& Cimini Jr, L.J. (1997). Effects of Clipping and Filtering on the Performance of OFDM, Proc. IEEE VTC 1997, pp. 1634-1638

Lloyd, S. (2006). Challenges of Mobile WiMAX RF Transceivers, Proceedings of the 8th International Conference on Solid-State and Integrated Circuit Technology, pp. 18211824, ISBN 1424401607, October, 2006, Shanghai

May, T. \& Rohling, H. (1998). Reducing the Peak-to-Average Power Ratio in OFDM Radio Transmission Systems, Proc. IEEE Vehicular Technology Conf. (VTC'98), pp.27742778

Mattsson, A.; Mendenhall, G. \& Dittmer, T. (1999). Comments on "Reduction of peak-toaverage power ratio of OFDM systems using a companding technique", IEEE Transactions on Broadcasting, Vol. 45, No. 4, pp. 418-419

Müller, S.H. \& Huber, J.B. (1997a). OFDM with Reduced Peak-to-Average Power Ratio by Optimum Combination of Partial Transmit Sequences, Electronics Letters, Vol.33, No.5, pp.368-369

Müller, S.H. \& Huber, J.B. (1997b). A Novel Peak Power Reduction Scheme for OFDM, Proc. IEEE PIMRC' '97, Helsinki, Finland, pp.1090-1094 
O'Neill, R. \& Lopes, L.B. (1995). Envelope variations and Spectral Splatter in Clipped Multicarrier signals, Proc. IEEE PIMRC '95, Toronto, Canada. pp. 71-75

Paterson, G.K. and Tarokh, V. (2000). On the Existence and Construction of Good Codes with Low Peak-to-Average Power Ratios, IEEE Transactions on Information Theory, Vol.46, No.6, pp. 1974-1987

Pauli, M \& Kuchenbecker, H.P. (1996). Minimization of the Intermodulation Distortion of a Nonlinearly Amplified OFDM Signal, Wireless Personal Communications, Vol.4, No.1, pp. 93-101

Sklar, B. (2001). Digital Communications - Fundamentals and Applications, 2nd Ed, Pearson Education, pp. 851-854

Stewart, B.G. \& Vallavaraj, A. 2008. The Application of $\mu$-Law Companding to the WiMax IEEE802.16e Down Link PUSC, 14th IEEE International Conference on Parallel and Distributed Systems, (ICPADS'08), pp. 896-901, Melbourne, December, 2008

Tarokh, V. \& Jafarkhani, H. (2000). On the computation and Reduction of the Peak-to-Average Power Ratio in Multicarrier Communications, IEEE Transactions on Communications, Vol.48, No.1, pp. 37-44

Tellambura, C. \& Jayalath, A.D.S. (2001). PAR reduction of an OFDM signal using partial transmit sequences, Proc. VTC 2001, Atlanta City, NJ, pp.465-469

Vallavaraj, A. (2008). An Investigation into the Application of Companding to Wireless OFDM Systems, PhD Thesis, Glasgow Caledonian University, 2008

Vallavaraj, A.; Stewart, B.G.; Harrison, D.K. \& McIntosh, F.G. (2004). Reduction of Peak-toAverage Power Ratio of OFDM Signals Using Companding, 9th Int. Conf. Commun. Systems (ICCS), Singapore, pp. 160-164

Van Eetvelt, P.; Wade, G. \& Tomlinson, M. (1996). Peak to average power reduction for OFDM schemes by selective scrambling, IEE Electronics Letters, Vol.32, No.21, pp. 1963-1964

Van Nee, R. \& De Wild, A. (1998). Reducing the peak-to-average power ratio of OFDM, Proc. IEEE Vehicular Technology Conf. (VTC'98), pp. 2072-2076

Van Nee, R. \& Prasad, R. (2000). OFDM for Wireless Multimedia Communications, Artech House, London, pp. 241-243

Wang, L. \& Tellambura, C. (2005). A Simplified Clipping and Filtering Technique for PAR Reduction in OFDM Systems, IEEE Signal Processing Letters, Vol.12, No.6, pp. 453456

Wang, L. \& Tellambura, C. (2006). An Overview of Peak-to-Average Power Ratio Reduction Techniques for OFDM Systems, Proc. IEEE International Symposium on Signal Processing and Information Technology, ISSPIT-2006, pp. 840-845

Wang, X., Tjhung, T.T. and Ng, C.S. (1999). Reduction of Peak-to-Average Power Ratio of OFDM System Using a Companding Technique, IEEE Transactions on Broadcasting, Vol.45, No.3, pp. 303-307

Yang, K. \& Chang, S.-II. (2003). Peak-to-Average Power Control in OFDM Using Standard Arrays of Linear Block Codes, IEEE Communications Letters, Vol.7, No.4, pp. 174-176 


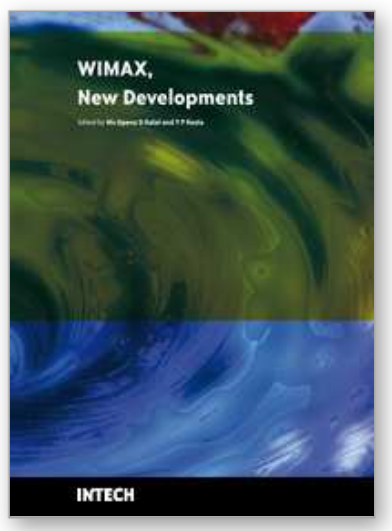

\author{
WIMAX New Developments \\ Edited by Upena D Dalal and Y P Kosta
}

ISBN 978-953-7619-53-4

Hard cover, 442 pages

Publisher InTech

Published online 01, December, 2009

Published in print edition December, 2009

WiMAX (Worldwide Interoperability for Microwave Access) is a wireless broadband access network named by industry group called the WiMAX forum formed in June 2001. It is Wireless MAN with IEEE 802.16 family standards. Loosely, WiMAX is a standardized wireless version of Ethernet that enables the last mile, intended primarily as an alternative to wire technologies (such as Cable Modems, DSL and T1/E1 links ) to provide broadband access to customer premises. Mission of the WiMAX forum is to promote and certify compatibility and interoperability of broadband wireless products. This book touches most of the above issues in form of 22 individuals' papers containing research work in WiMAX domain in particular. WiMAX has two important standards/usage models: a fixed usage model IEEE 802.16-2004 for Fixed Wireless Broadband Access (FWBA) and a portable usage model IEEE 802.16e-2005, which is mainly concentrated on Mobile Wireless Broadband Access (MWBA). Both are released as standards and amendments are available in form of drafts. Higher data rate transmissions (@ 100 Mbps) are achieved in IEEE 802.16-2004 WiMAX through LOS communications which incorporate a stationary transmitter and receiver but IEEE 802.16e supporting NLOS communication is much complicated and little less bit rate is achieved. $2-11 \mathrm{GHz}$ licensed band is the range of frequencies with TDD and FDD supports. The book will provide a wide horizon to visualize the WiMAX technology and its developments leading towards $4 \mathrm{G}$ systems. It will provide a good platform to the researchers with clues to the innovative ideas in WiMAX domain. I wish all the best to the authors and readers of this book in their successful research of WiMAX technology.

\title{
How to reference
}

In order to correctly reference this scholarly work, feel free to copy and paste the following:

Brian G Stewart and Athinarayanan Vallavaraj (2009). The Application of -Law Companding to Mobile WiMax, WIMAX New Developments, Upena D Dalal and Y P Kosta (Ed.), ISBN: 978-953-7619-53-4, InTech, Available from: http://www.intechopen.com/books/wimax-new-developments/the-application-of-law-companding-tomobile-wimax

\section{INTECH}

open science | open minds

\section{InTech Europe}

University Campus STeP Ri

Slavka Krautzeka 83/A

51000 Rijeka, Croatia

Phone: +385 (51) 770447

\section{InTech China}

Unit 405, Office Block, Hotel Equatorial Shanghai

No.65, Yan An Road (West), Shanghai, 200040, China

中国上海市延安西路65号上海国际贵都大饭店办公楼 405 单元

Phone: +86-21-62489820 
Fax: +385 (51) 686166

Fax: +86-21-62489821

www.intechopen.com 
(C) 2009 The Author(s). Licensee IntechOpen. This chapter is distributed under the terms of the Creative Commons Attribution-NonCommercial-ShareAlike-3.0 License, which permits use, distribution and reproduction for non-commercial purposes, provided the original is properly cited and derivative works building on this content are distributed under the same license. 